\title{
CONFLITO ENTRE ECONOMIA E SAÚDE? O CASO DA COVID-19 NO BRASIL
}

\section{CONFLICT BETWEEN ECONOMY AND HEALTH? THE CASE OF COVID-19 IN BRAZIL}

\author{
EDGAR REYES JUNIOR \\ Professor do PPG em Administração da Universidade de Brasília - PPGA/UnB \\ Doutor em Administração (UNISINOS)
}

Orcid: https://orcid.org/0000-0001-7403-5909 / E-mail: prof.edreyes@gmail.com Campus Universitário Darcy Ribeiro, Brasília-DF

\section{FAGNER DE OLIVEIRA DIAS}

Doutorando em Administração com ênfase em Estratégia Organizacional - UnB PPG em Administração da Universidade de Brasília - PPGA/UnB

Orcid: https://orcid.org/0000-0001-6529-5485 / E-mail: fagner.pmdf@gmail.com

\section{CAROLINE CORDOVA BICUDO DA COSTA}

Doutoranda em Administração com ênfase em Estratégia Organizacional - UnB PPG em Administração da Universidade de Brasília - PPGA/UnB

Orcid: https://orcid.org/0000-0003-3251-0059 / E-mail: carolinecordova@live.com

\author{
FLAVIO SAAB \\ Doutorando em Administração com ênfase em administração pública e políticas públicas - UnB \\ PPG em Administração da Universidade de Brasília - PPGA/UnB \\ Orcid: https://orcid.org/0000-0002-3913-4175 / E-mail: flaviosaab@gmail.com
}

\section{ELOISA GONÇALVES DA SILVA TORLIG}

Doutoranda em Administração com ênfase em administração pública e políticas públicas - UnB PPG em Administração da Universidade de Brasília - PPGA/UnB

Orcid: https://orcid.org/0000-0003-2519-9805 / E-mail: eloisatorlig@gmail.com

Submissão: 15/05/2020. Revisão: 20/08/2020. Aceite: 05/11/2020. Publicação: 29/12/2020. DOI: http://dx.doi.org/10.22277/rgo.v14i1.5486

\section{RESUMO}

Com a ocorrência da pandemia mundial da COVID-19 foi observado no Brasil um aparente conflito entre economia e saúde, gerando problemas na administração pública para uma tomada de decisão eficaz. O presente artigo propõe uma estrutura de gestão estratégica de primeira resposta a problemas complexos e inesperados no setor público para o reconhecimento do problema e resposta eficiente, baseada no modelo estratégico em desastres e na estrutura da complexidade institucional. Como metodologia, utilizou-se a combinação sistemática da lógica abdutiva por meio da análise de notícias de jornal, analisando como os conflitos eram explicitados por meio da Classificação Hierárquica Descendente (CHD), Análise Fatorial por Correspondência (AFC) e análise do conteúdo da notícia. Como resultados observam-se: 1. A existência de 5 divisões de discurso, Pandemia, Legislativo, Pluralidade Social, Resposta Federal e Conflito; 2. A evidenciação de todas as fases iniciais (complexidade institucional, incerteza técnico-científica, falha na liderança e pluralismo social); 3 . A origem do conflito não se encontrava entre economia e saúde, mas sim em um conflito político entre o governo federal e os governadores; 4 . Saúde e economia foram somente ferramentas neste embate.

Palavras-chave: Gestão Estratégica. Complexidade Institucional. Primeira Resposta. Setor Público. COVID-19. 


\begin{abstract}
With the occurrence of the global pandemic of COVID-19, the apparent conflict between economy and health was observed in Brazil, creating problems in public administration for effective decision making. This article proposes a strategic management structure of first response to complex and unexpected problems in the public sector for the recognition of the problem and efficient response, based on the strategic model in disasters and the structure of institutional complexity. As a methodology, we used the systematic combination of abductive logic through the analysis of newspaper news, analyzing how conflicts were made explicit through the Descending Hierarchical Classification (CHD), Factorial Analysis by Correspondence (AFC) and analysis of the news content. As results we can observe: 1) The existence of 5 discourse divisions, Pandemic, Legislative, Social Plurality, Federal Response and Conflict; 2. the disclosure of all the initial phases (institutional complexity, technical-scientific uncertainty, failure in leadership and social pluralism). 3. the source of the conflict was not found between economics and health, but in a political conflict between the federal government and the governors. 4. that health and economy were only tools in this struggle.
\end{abstract}

Keywords: Strategic management. Conflict. Institutional complexity. Public sector. COVID-19.

\title{
1 INTRODUÇÃO
}

Recentemente, o mundo presenciou um grande surto de doença que mudou a rotina de toda a população, a pandemia do novo coronavírus - COVID-19. Estudos anteriores ao ocorrido atualmente já haviam percebido, em uma análise de políticas de preparação para pandemia nas nações europeias, a existência de uma necessidade de reforçar o sistema de controle para prevenir e conter pandemias que não haviam sido adotadas ainda (KRUMKAMP et al., 2009). A diversidade de opções e níveis de gerenciamento de ações de combate a uma pandemia, se não reconhecida e identificada corretamente, pode gerar dúvidas nos tomadores de decisão do setor público. Por esse motivo a necessidade de um modelo de gestão estratégica com foco no reconhecimento do problema para posterior resposta organizacional abordado neste estudo.

Nos estudos sobre os impactos econômicos das medidas de mitigação do vírus influenza, como exemplo de comparação, verificou-se que todas as medidas de distanciamento social diminuíam a propagação do vírus, porém ações de melhor efetividade como o fechamento de escolas e a profilaxia antiviral direcionada custaram vinte vezes mais que as ações menos incisivas (SANDER et al., 2009). Essa decisão entre qual medida tomar e seu impacto econômico são métricas levadas em consideração quanto às tomadas de decisão no Setor Público.

No Brasil, jornais e redes virtuais têm exaltado o debate sobre as decisões de isolamento e distanciamento social e sugerem um antagonismo entre economia e saúde e seus impactos na população, no qual os impactos econômicos poderiam gerar problemas ainda maiores no país do que a própria pandemia, enquanto de outro lado defende-se que a redução da propagação do vírus é necessária para reduzir a curva de contágio e não sobrecarregar o sistema de saúde. Essa suposta dicotomia vem gerando incertezas e, consequentemente, diversidades de ações das autoridades envolvidas que implicam em dificuldades na resposta do setor público para um problema complexo devido à falta de conhecimento completo do problema. 
Edgar Reyes Junior, Fagner de Oliveira Dias, Caroline Cordova Bicudo da Costa, Flávio Saab e Eloisa Gonçalves da Silva Torlig

Nesse contexto, surge a necessidade de um modelo de gestão estratégica em casos de Problema Público Complexo e Inesperado - $\mathrm{PPCl}$, no qual os gestores e tomadores de decisão possam compreender e identificar o real problema e se nortear para analisar o cenário e propor as melhores respostas frente aos problemas identificados. O reconhecimento desse aspecto em momentos de pandemia pode reduzir o tempo de resposta e os impactos negativos causados pela morosidade de uma ação efetiva.

Dessa forma, o presente artigo pretende responder a seguinte questão de pesquisa: $E$ realmente o conflito entre economia e saúde que impacta na tomada de decisão da administração pública na pandemia de COVID-19?

Para responder a essa pergunta propomos um modelo de gestão estratégica em problemas complexos e inesperados no Setor Público, que se baseia em uma análise de quatro dimensões de conflitos iniciais de problemas complexos, já utilizada em início de desastres industriais e que tem como base a complexidade institucional. Utilizar os parâmetros iniciais de conflito institucional, juntamente com a identificação do ciclo dimensional de conflitos e com as proposições da resposta organizacional, pode ser um modelo promissor para a administração pública.

\section{REFERENCIAL TEÓRICO}

\subsection{LÓGICA, COMPLEXIDADE E RESPOSTA ORGANIZACIONAL}

A lógica institucional surge como parte do desenvolvimento da teoria institucional desde os anos 70, em que pode ser vista como uma abordagem metateórica e um método de análise das instituições (THORNTON; OCASIO, 2008). A lógica institucional procura fazer a ligação entre instituições e ações, abordando uma ponte entre a perspectiva estrutural macro de Meyer e Rowan (1977) e Dimaggio e Powell (1983) e a abordagem de processos micro de Zucker (1977). Assim, as lógicas institucionais podem ser definidas como os padrões históricos socialmente construídos de práticas materiais, suposições, valores, crenças e regras pelos quais os indivíduos dão significado a sua realidade social (THORNTON; OCASIO, 1999).

Em suma, a lógica institucional é aquilo que fornece diretrizes organizacionais sobre como compreender e agir em situações sociais. As organizações seguem a lógica para serem aprovadas e legitimadas pelo público de referência e por dar um significado à compreensão do contexto social (GREENWOOD et al., 2011). Essa lógica acaba por ser o resultado do ajuste entre as normas e as relações sociais de uma organização que orientam sua forma de agir. A lógica institucional serve como base para aprofundar o conhecimento das relações entre indivíduos, organizações e sociedade (FRIEDLAND; ALFORD, 1991).

Quando, em um campo organizacional, uma organização enfrenta prescrições incompatíveis de várias lógicas institucionais ela se depara com a complexidade institucional (GREENWOOD et al., 2011), ou seja, o conflito entre lógicas. Uma organização pública como a escola, por exemplo, tem a educação sujeita a diferentes lógicas, os pais veem de uma forma, os professores de outra, o governo de outra, e esse conflito pressupõe uma resposta da escola que deve se adaptar para suprir o conflito. Outro caso de conflito de lógicas surge nas multinacionais que abrem uma nova sede em outro país e têm um conflito entre a lógica empresarial e a lógica local. Para compreender a magnitude desse conflito a estrutura do campo é essencial. Essa pode ser compreendida pela fragmentação, formalização e centralização no campo (GREENWOOD et al., 2011).

A fragmentação refere-se aos constituintes não coordenados necessários para a legitimidade de uma organização, em que a coexistência desses atores favorece conjuntos 
díspares de prescrições institucionais que pressionam a organização (PACHE; SANTOS, 2010). No exemplo anterior, sobre uma escola pública, a fragmentação está na necessidade de legitimação da educação pelos pais, professores, poder público e dos próprios alunos, entre outros. No momento em que uma atividade educacional vai de encontro à prescrição institucional dos pais de alunos, por exemplo, mesmo que aprovada pelo governo e pelo corpo discente, não se legitima totalmente, gerando a complexidade institucional.

A formalização refere-se ao aspecto da constituição formal ou informal das demandas organizacionais, demandas menos organizadas causam menos pressão institucional, porém as organizações mais formalizadas podem aumentar a especificidade e responder de forma mais calculável, dessa forma, o real impacto da formalização na complexidade é, ainda, empiricamente desconhecido (GREENWOOD et al., 2011).

A centralização diz respeito a estrutura hierárquica de poder das instituições constituídas, como exemplo a centralização no campo da educação das escolas sob a mesma égide padronizam as formas educacionais e diminuem a complexidade (MEYER; SCOTT; STRANG, 1987), assim a centralização por meio da hierarquia de poder transfere as demandas institucionais a níveis mais altos que diminuem a complexidade na organização (GREENWOOD et al., 2011). Assim, escolas que estão subordinadas à hierarquia tendem a sofrer menos pressão institucional, concentrando essas demandas nos níveis mais altos do campo hierarquizado.

No setor público brasileiro, por exemplo, a fragmentação e a centralização são elementos claramente presentes. O poder executivo se estrutura em hierarquia de poder que foca as demandas nos poderes mais altos para reduzir pressões institucionais nas organizações e, por outro lado, necessita ser legitimado tanto por atores no âmbito ideológico, de mercado e científico, e quando existem diferenças nas prescrições destas lógicas o conflito é instaurado. Dessa forma, as pressões institucionais acabam por focar nos tomadores de decisão que estão representando o mais alto nível hierárquico do campo da administração pública, o poder executivo, que tem sua legitimidade fragmentada por fatores ideológicos, técnicos, econômicos entre outros, que sugerem constante complexidade institucional.

Assim, a experiência com complexidade institucional exige uma resposta da organização para esses conflitos. Os atributos da organização são moderadores dessa resposta, tornando-a mais fácil ou difícil, esses se subdividem em: posição no campo, estrutura, governança e identidade (GREENWOOD et al., 2011). Se uma organização tem posição de superioridade no campo, a maior obrigação de se remodelar passa a ser da outra. Quanto maior a estrutura de uma organização, mais difícil de alterar sua lógica. Se a governança for mais centralizada, haverá menor resistência na mudança. E por fim, quanto mais a resposta organizacional "ferir" a identidade da organização, maior será a dificuldade em aceitar tal mudança.

Os líderes da organização são os atores que respondem às pressões institucionais por necessitarem explicar aos observantes externos que as escolhas feitas são apropriadas (PACHE; SANTOS, 2010). Logo, as respostas organizacionais são as ações e escolhas dos tomadores de decisão buscando alcançar os objetivos organizacionais. Para Greenwood et al. (2011, p.319), em casos de complexidade, as respostas organizacionais "são importantes porque podem ter grandes implicações para legitimidade social e, portanto, para o acesso de uma organização e recursos críticos". Percebe-se como fundamental a compreensão do conflito e a procura pela melhor maneira de se responder a ele. 
Edgar Reyes Junior, Fagner de Oliveira Dias, Caroline Cordova Bicudo da Costa, Flávio Saab e Eloisa Gonçalves da Silva Torlig

\subsection{GESTÃO ESTRATÉGICA DE PROBLEMAS PÚBLICOS COMPLEXOS}

Falar em gestão estratégica implica em abranger as decisões possíveis tomadas por organizações que devem se posicionar, não apenas em seu cenário competitivo ou mercado de recursos, mas também em relação às lógicas institucionais (DURAND, 2012). A maior parte da literatura em gestão estratégica no setor público trata de problemas esperados, porém são necessárias diretrizes para problemas inesperados e incomuns.

Problemas complexos e inesperados, também chamados de Wicked Problems, são aqueles compostos por três aspectos: não são estruturados porque são difíceis de formular e mudam a cada tentativa de solução; a existência de vários subconjuntos e sobreposições de problemas que refletem hierarquias e estruturas de autoridade dentro e entre organizações, domínios de políticas, jurisdições e partes interessadas e; são problemas implacáveis que não serão resolvidos de uma vez por todas mesmo com boas intenções e recursos direcionados (WEBER; KHADEMIAN, 2008).

Em sua proposta de estrutura analítica para governança colaborativa e adaptativa para lidar com o Wicked Problem, mais especificamente com os desastres, Abreu e Andrade (2019) propõem um modelo em três níveis para lidar com desastres industriais, sendo eles: 1) Melhoria de Políticas, que compreende entender o problema e responder com Governança Pública; 2) Aprendendo Reestruturando, que consiste em se reestruturar baseado em uma Governança Colaborativa e; 3) Aprendendo Transformando, que consiste no processo de aprendizado e resiliência com uma Governança Adaptativa. Porém, para uma análise da primeira resposta do setor público, conforme escopo deste estudo, interessa analisar o primeiro nível da estrutura analítica.

Conforme os autores, o primeiro nível diz respeito às quatro características cíclicas que ocorrem imediatamente após o desastre industrial e que trabalham juntas para aumentar o nível de complexidade, incerteza e disputa. Inicialmente ocorre a complexidade institucional, ou seja, os conflitos de lógicas das organizações envolvidas. A natureza e extensão da complexidade são moldadas pela incerteza técnico-científica que reflete as divergências nas diferentes estratégias das instituições envolvidas. Um cenário de reações lentas e prematuras leva a situações de conflito e uma falha na liderança, caracterizada por custos, incertezas, dependência de caminho e falta de poder e legitimidade. Por fim, afloram os interesses segmentados dos indivíduos, ou pluralismo social, pois os indivíduos se veem sujeitos a pressões conflitantes, que satisfazem uma área do conjunto de ideias politicamente relevantes, mas que também geram insatisfações, podendo gerar impulsos políticos contrários.

\subsection{MODELO DE GESTÃO ESTRATÉGICA DE PRIMEIRA RESPOSTA NO SETOR PÚBLICO}

Com a finalidade de propor um modelo de primeira resposta no setor público ao Wicked Problem, que aqui será chamado de Problemas Públicos Complexos e Inesperados $\mathrm{PPCl}$, foi utilizado o primeiro nível da estrutura analítica de desastre industrial de forma genérica, para qualquer $\mathrm{PPCl}$ juntamente com a estrutura de análise de resposta organizacional à complexidade institucional.

Conforme apresentado na Figura 1, quando da ocorrência do $\mathrm{PPCl}$ no setor público, que é plurinstitucional, haverá naturalmente um conflito de lógicas institucionais que terá intensidade conforme a estrutura do campo. A complexidade institucional, por suas características de divergências institucionais, proporcionará diferentes soluções para o problema gerando incerteza técnico-científica. Essa incerteza causa demora na resposta e 
situações de conflito que denotam a falha de liderança. Depois desses conflitos instaurados, emergem os interesses de indivíduos ou grupos conforme cada conjunto de ideias gerando a pluralidade social que reforça ainda mais a complexidade institucional.

Para dar a primeira resposta à complexidade institucional instaurada, é necessário modificar lógicas institucionais, que terá seu nível de dificuldade mensurado conforme atributos das organizações envolvidas no conflito. Por fim, propomos quatro ações de primeira resposta baseados no primeiro estágio de Abreu e Andrade (2019), que devem ser estruturadas após a compreensão dos atributos organizacionais para conter os quatro estágios de conflito. Os quatro direcionamentos de primeira resposta são: comando e controle; informação especializada; liderança hierárquica e; redes invisíveis.

O comando e controle tem por finalidade centralizar a tomada de decisão e reduzir a autonomia das organizações envolvidas na complexidade institucional nivelando hierarquias, sendo importante que as partes envolvidas concordem com a transferência de poder (ROBERT, 2000). A informação especializada é indicativa de solução convergente, conforme o paradigma hegemônico (KUHN, 2012), reduzindo a incerteza técnico-científica. A liderança hierárquica define a ordem de tomada de decisão e a organização "chave", facilitando no apoio e recursos (HERNANTES; BAÑULS, 2017) reduzindo a falha de liderança. E as redes invisíveis, além de serem mais livres para pensar em políticas públicas alternativas (FOLKE, 2006), procuram mitigar o pluralismo social apaziguando os ânimos através de atores externos não evidenciados no conflito. Assim, os conflitos iniciais são mitigados para a adoção das fases posteriores de resolução do problema e retomada da estabilização social.

Figura 1 - Modelo de Gestão Estratégica de Primeira Resposta no Setor Público

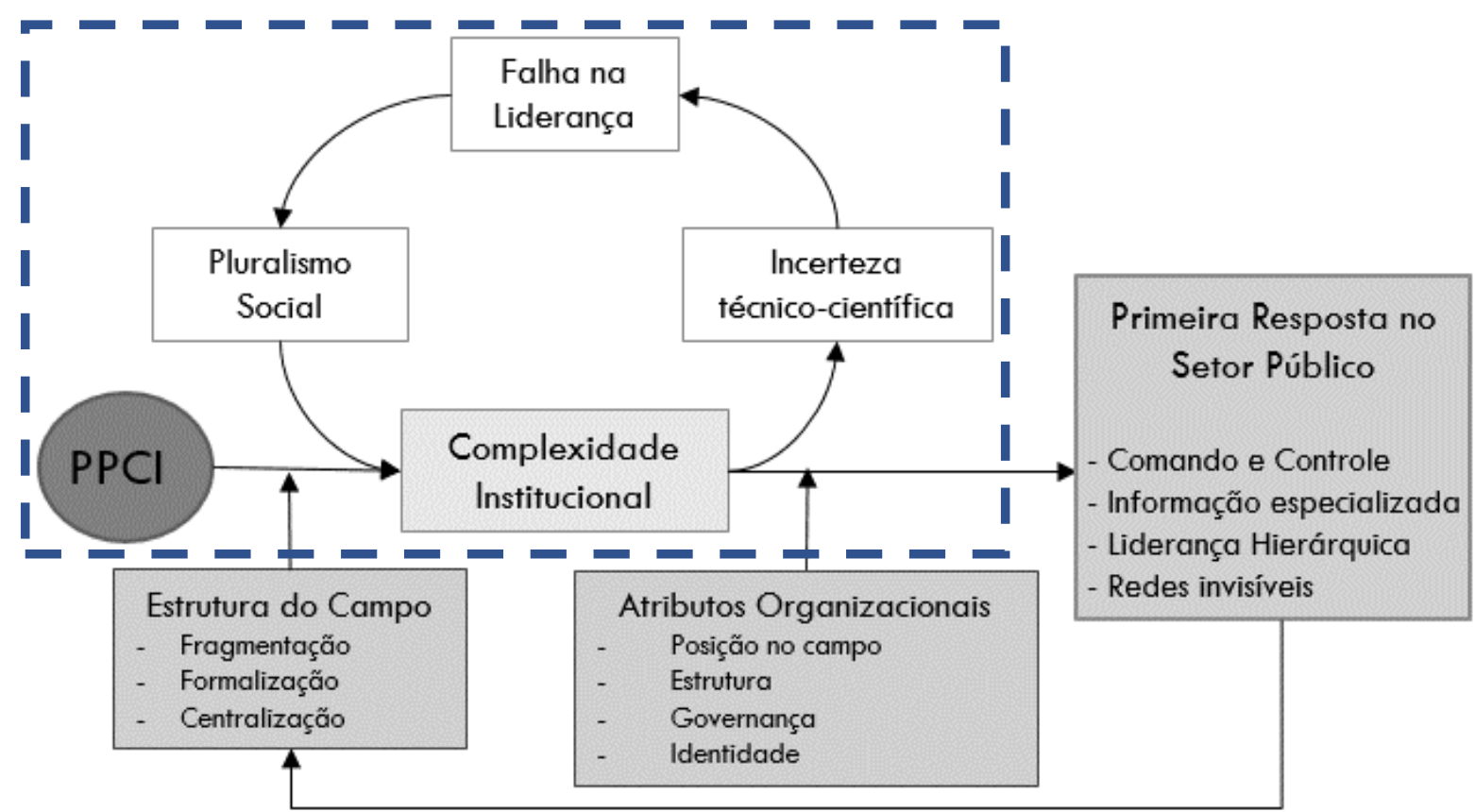

Fonte: adaptado de Abreu e Andrade (2019) e Greenwood et al. (2011).

Este trabalho se aterá à parte do modelo correspondente à análise do ciclo do conflito, conforme área tracejada, que se inicia na complexidade institucional gerada pela COVID-19, pelo fato de que essa parte do modelo representa a capacidade de reconhecer a real causa do conflito, fator importante para as sugestões e análises futuras que envolvem a resposta organizacional, além do fato da pandemia ainda se encontrar em curso, não havendo clareza, 
Edgar Reyes Junior, Fagner de Oliveira Dias, Caroline Cordova Bicudo da Costa, Flávio Saab e Eloisa Gonçalves da Silva Torlig

no período analisado, dos ciclos de primeira resposta dados e de sua influência na estrutura do campo.

\section{MÉTODO}

A pesquisa adotou uma abordagem baseada na combinação sistemática de uma lógica abdutiva que é particularmente adequada para a compreensão de pesquisas de caso único destinada ao desenvolvimento de teoria (DUBOIS; GADDE, 2002). Ainda segundo os autores, a combinação sistemática consiste na combinação de esforços entre estrutura teórica, trabalho empírico e a análise de estudo de caso que evoluem em conjunto. Enfatiza-se aqui que a combinação sistemática se baseia no refinamento de teorias existentes com foco na estrutura, diferente de estudos dedutivos.

Logo, para realizar a análise da proposição teórica e do caso em questão, foi realizada uma verificação das publicações de notícias com o intuito de buscar acontecimentos que corroborem com o modelo apresentado. A coleta de dados se referiu à busca por notícias no site da Folha de São Paulo, no período entre 26 de fevereiro e 03 de abril de 2020 e com os filtros de editoriais "poder" e "equilíbrio e saúde". Optou-se pela Folha de São Paulo por tratar-se do jornal de maior circulação no Brasil, o que confere a esse periódico melhor representação dos fatos e notícias veiculadas no país. A escolha do recorte temporal objetivou entender o desenvolvimento da resposta governamental à situação de pandemia de COVID19 entre o reconhecimento do primeiro caso da doença no país pelo Ministério da Saúde e a decretação de calamidade pelo Congresso Nacional. A busca considerou 3 conjuntos de palavras-chave que envolveram a doença e atores do Poder Executivo envolvidos em áreas críticas como a Presidência, Ministério da Saúde e Ministério da Economia, quais sejam: "Bolsonaro" e "COVID-19"; "Guedes" e "COVID-19" e; "Mandetta" e "COVID-19", tendo sido retornado o total de 280 notícias. Seguindo o critério de necessidade de explicitação de um conflito de ideias, visão ou opinião entre atores dentro do contexto da pandemia, 82 notícias foram consideradas aptas ao estudo. Como último passo, precedeu-se com a exclusão de notícias duplicadas, resultando em 65 notícias o corpus textual.

Após organizados, os dados foram analisados por meio do software IRAMUTEQ, utilizando-se a palavra como unidade de registro, conforme critérios semânticos. Foram definidas a priori quatro categorias de interesse que representam as quatro características cíclicas do primeiro nível do modelo proposto: complexidade institucional, incerteza técnicocientífica, falha na liderança e pluralismo social. Para proceder com a divisão e classificação dos segmentos de texto presentes no corpus textual conforme vocábulos associados realizouse a Classificação Hierárquica Descendente (CHD), análise que parte da lógica de correlação para apresentar um esquema hierárquico de classes (CAMARGO; JUSTO, 2013). A dimensão da associação das palavras do corpus textual a cada classe pode ser verificada por meio do valor de seus respectivos $\chi^{2}$, sendo que a significância se inicia com o valor de $\chi^{2}=3,80$ (SALVIATI, 2017). Após a realização da CHD, foi possível acessar a Análise Fatorial por Correspondência (AFC), que representou em um plano cartesiano as palavras associadas a cada classe, possibilitando a análise mais qualitativa dos dados.

Seguindo os passos sugeridos por Bardin (1977), após a fase inicial de divisão e classificação textual, procedeu-se com a codificação de segmentos de texto que apresentaram as unidades de registro associadas às classes em que foram observadas situações de divergências de lógica, em alinhamento ao modelo proposto. Foi realizada a interpretação do conteúdo de tais segmentos de texto a partir do entendimento do significado das palavras mais representativas do contexto de cada categoria de interesse. 
Finalmente, para representar a evidência empírica associada a cada categoria, foram selecionados, entre os mais representativos, quatro segmentos de texto que apresentaram ao menos duas palavras classificadas entre as quinze mais significativas de cada classe de interesse, conforme respectivo valor de $\chi^{2}$. A opção pela utilização das análises descritas baseou-se no interesse de entender quais são e como se organizam os diferentes contextos da pandemia de COVID-19 no país.

\section{ANÁLISE E DISCUSSÃO DOS RESULTADOS}

Ao realizar a CHD, conforme Figura 2, observou-se uma separação das principais palavras em cinco grupos denominados Pandemia, Legislativo, Pluralidade Social, Resposta Federal e Conflito. Pandemia trata da apresentação de dados da COVID-19 no mundo, com a apresentação de dados técnicos sobre a doença. Aqui se destacam palavras como "caso", "paciente" e "vírus". Legislativo apresenta o processo de interação entre os poderes executivo e legislativo, cujas palavras principais remetem à ação no parlamento e suas ações para a mitigação da pandemia. São observadas as palavras "executivo", "orçamento" e "votação". Ambas as divisões apresentam objetividade ou tecnicidade demonstrando a racionalidade na discussão apresentada.

O campo Resposta Federal descreve os principais atores do executivo federal e os elementos-chave que permitem a compreensão do caráter político do conflito. Salientam-se palavras como ministro, Bolsonaro, ideológico e aliado. Conflito apresenta os oponentes do executivo federal e as principais ferramentas do embate entre eles. Observam-se os termos governador, histeria, emprego e criticar. Salienta-se que esta divisão é a única que contém elementos emocionais em seus discursos, o que caracteriza o estabelecimento do efetivo conflito. Para a análise de complexidade institucional fica, assim, identificado o conflito entre as lógicas do governo federal e as lógicas dos governos estaduais.

Figura 2 - Representação da Classificação Hierárquica Descendente

\begin{tabular}{|c|c|c|c|c|c|c|c|c|c|}
\hline $\begin{array}{c}\text { Pandemia } \\
28,2 \%\end{array}$ & $x^{2}$ & $11,2 \%$ & $\chi^{2}$ & $\begin{array}{c}\text { Pluralidade } \\
\text { Social } \\
13,4 \% \\
\end{array}$ & $\chi^{2}$ & $\begin{array}{c}\text { Resposta } \\
\text { Federal } \\
19,4 \% \\
\end{array}$ & $\chi^{2}$ & $\begin{array}{c}\text { Conflito } \\
27,8 \%\end{array}$ & $\chi^{2}$ \\
\hline \multirow{5}{*}{$\begin{array}{r}\text { caso } \\
\text { confirmar } \\
\text { número } \\
\text { paciente } \\
\text { itália }\end{array}$} & 215,9 & \multirow{5}{*}{$\begin{array}{r}\text { executivo } \\
\text { orçamento } \\
\text { legislativo } \\
\text { real } \\
\text { câmara }\end{array}$} & 145,2 & \multirow{3}{*}{$\begin{array}{r}\text { manifestação } \\
\text { favor } \\
\text { aglomeração }\end{array}$} & 177,6 & \multirow{5}{*}{$\begin{array}{r}\text { ministro } \\
\text { bolsonaro } \\
\text { anvisa } \\
\text { integrante } \\
\text { último }\end{array}$} & 109,4 & \multirow{3}{*}{$\begin{array}{r}\text { governador } \\
\text { histeria } \\
\text { emprego }\end{array}$} & 57,6 \\
\hline & 93,9 & & 127,4 & & 83,9 & & 78,5 & & 47,3 \\
\hline & 81,6 & & 114,4 & & 81,8 & & 44,5 & & 42,8 \\
\hline & 55,3 & & 112,4 & ato & 73,5 & & 41,7 & vida & 41,3 \\
\hline & 43,9 & & 87,05 & participar & 67,8 & & 38,5 & criticar & 39,1 \\
\hline viagem & 42,2 & senado & 71,9 & apoiador & 65,8 & sérgio moro & 36,8 & pronunciamento & 38,3 \\
\hline país & 41,4 & relator & 71,9 & protesto & 59,3 & mandetta & 36,3 & população & 31,8 \\
\hline critério & 41,2 & casa civil & 71,9 & jornalista & 52,3 & ideológico & 36,2 & economia & 30,1 \\
\hline vírus & 40,5 & votação & 70,9 & evitar & 51,05 & ação & 35,6 & fechamento & 29,7 \\
\hline mundo & 38,7 & congressista & 70,7 & coletivo & 50,5 & discurso & 35,04 & isolado & 28,8 \\
\hline sintoma & 36,7 & recurso & 67,7 & manifestante & 47,1 & planalto & 33,2 & prefeito & 27,3 \\
\hline china & 33,4 & maia & 66,9 & redes sociais & 44,9 & torres & 31,9 & importância & 25,6 \\
\hline gente & 33,2 & partidário & 63,8 & recomendar & 44,8 & governo & 31,6 & desemprego & 25,2 \\
\hline morrer & 30,6 & projeto & 63,4 & som & 39,09 & aliado & 27,9 & decreto & 25,2 \\
\hline doença & 29,8 & congresso & 60,5 & ignorar & 38,6 & tornar & 27,9 & onda & 24,6 \\
\hline
\end{tabular}

Fonte: elaborada pelos autores (2020). 
Edgar Reyes Junior, Fagner de Oliveira Dias, Caroline Cordova Bicudo da Costa, Flávio Saab e Eloisa Gonçalves da Silva Torlig

Por fim, Pluralidade Social, apresenta a consequência do conflito estabelecido entre Resposta Federal e Conflito. Destacam-se expressões como manifestação, aglomeração e protesto. Apesar de existir uma relação de proximidade entre Resposta Federal e Pluralidade Social, não ficou evidenciado um conflito entre lógicas do governo federal, mantendo então o foco da análise na relação de conflito anterior.

Na AFC, conforme Figura 3, pode-se verificar a formação de 3 regiões distintas de distribuição de palavras. Os grupos Pandemia e Legislativo representados, respectivamente, em vermelho e roxo, encontram-se claramente isolados e em áreas independentes.

Pluralidade Social (verde), Resposta Federal (cinza) e Conflito (azul) encontram-se compartilhando o mesmo espaço, com o primeiro separando basicamente as demais. Essa representação confirma o estabelecimento do conflito, evidenciando que as notícias de reação pública estão ligadas tanto às respostas federais como aos conflitos de presidência e governo.

Figura 3 - Representação da Análise Fatorial por Correspondência

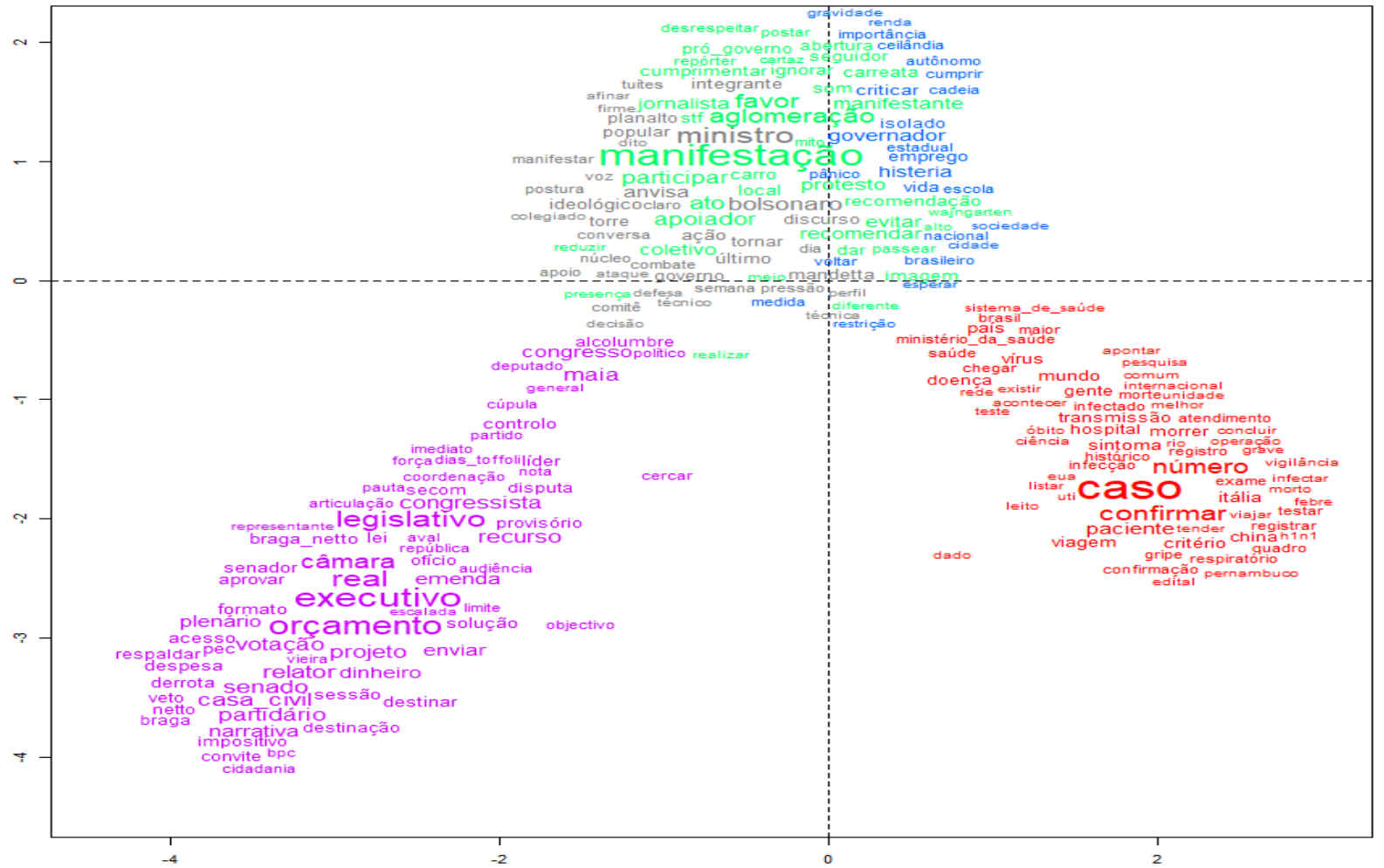

Fonte: elaborada pelos autores (2020).

Por meio da classificação dos segmentos de texto associadas às classes, identificou-se divergências de lógicas institucionais e os elementos da complexidade institucional do conflito.

A Complexidade Institucional (conflito entre a lógica do governo federal e do governo estadual) pode ser explicitada no trecho: "na entrevista, Bolsonaro voltou a falar que as ações de governadores prejudicam a economia e podem criar um ambiente de caos no país o que, segundo ele, pode gerar saques a supermercados e instabilidade democrática. Ele citou a esquerda e deu os protestos do Chile como exemplo". O trecho demonstra o conflito de práticas e comportamentos cotidianos indo de encontro aos critérios normativos de suas lógicas (DACIN; MUNIR; TRACEY, 2010), porém ao analisar o âmbito estrutura de campo, observa-se que os governos federal e estadual são representados por organizações formalizadas e centralizadas no quesito de tomada de decisão, sendo a fragmentação por 
legitimidade política, o principal causador da complexidade institucional, diferente do conflito esperado entre economia e saúde, corroborando com a afirmação que a fragmentação sozinha aumenta a complexidade que uma organização enfrenta (GREENWOOD et al., 2011).

Quanto à Incerteza Técnico-Científica, sua demonstração pode ser observada no conflito dos paradigmas da saúde e economia como instrumento para resolução do problema conforme o trecho: "ele diz que os idosos devem ser protegidos, mas que a maior parte da população poderia voltar à rotina. As declarações de Bolsonaro contrariam recomendações de autoridades médicas e os governadores decidiram ignorar os apelos de Bolsonaro para abrandar as restrições". Em momentos de um evidente conflito de paradigmas é necessário parar de se esquivar das anomalias e conduzir a um novo um paradigma hegemônico (KUHN, 2012)

A Falha na Liderança é mencionada no trecho:

Ele [Bolsonaro] voltou a criticar alguns governadores e prefeitos pelas restrições que impuseram em seus estados e municípios "esta neurose de fechar tudo não está dando certo". Na transmissão ao vivo, ele também se queixou indiretamente das críticas que recebeu da cúpula militar de seu governo.

Isso evidencia o conflito de lideranças, sendo um consequência a falta de ajuda estratégica, suporte e recursos (HERNANTES; BAÑULS, 2017) que atrasa a resolução dos problemas devido às incertezas geradas e falta de legitimidade e poder (ROSENSCHÖLD; ROZEMA; FRYE-LEVINE, 2014).

Por fim, quanto ao Pluralismo Social:

Bolsonaristas que concordam com o Bolsonaro convocaram protestos e carreatas pedindo a reabertura dos comércios e quarentena apenas para idosos. Bolsonaro compartilhou um vídeo em suas redes sociais com a frase "o povo quer trabalhar". Nas imagens, carros faziam uma carreata e buzinavam em Balneário Camboriú.

O trecho evidencia o interesse segmentado dos indivíduos que estão sujeitos a pressões conflitantes devido a falha de liderança (ABREU; ANDRADE, 2019) em um ambiente de conflitos políticos que utilizam de um suposto trade-off entre economia e saúde.

\section{CONSIDERAÇÕES FINAIS}

O presente estudo teve por objetivo analisar o impacto do conflito entre economia e saúde na tomada de decisão da administração pública na pandemia de COVID-19. São identificados os diferentes espaços de fala. Somente em Conflito se observa um discurso que envolve carga emocional identificando o caráter político do conflito estabelecido. Essa observação sugere que a origem do conflito não se encontrava entre economia e saúde, mas sim em um conflito político entre o governo federal e os governadores. Saúde e economia, que aparentavam ser os elementos do conflito, acabam por se caracterizar como meras ferramentas para este embate.

Teoricamente, as ocorrências nas diferentes fases do modelo, fortalecem a ideia de sua utilização em casos de crise, ressaltando-se que analisar a estrutura do campo permitiu compreender que o conflito não ocorria entre saúde e economia e sim entre governo federal e estaduais. Observou-se ainda que tal conflito se origina da necessidade de legitimação política de ambos, e que saúde e economia foram ferramentas para este embate. Em questões sociais, a devida compreensão dos fenômenos pode ser fundamental para a pesquisa científica, sem a qual não há como se chegar a respostas com suficiente capacidade explicativa. 
Edgar Reyes Junior, Fagner de Oliveira Dias, Caroline Cordova Bicudo da Costa, Flávio Saab e Eloisa Gonçalves da Silva Torlig

Quanto às limitações, observam-se que os dados foram coletados de apenas um jornal. Essa fonte única, mesmo com textos de diferentes redatores, pode trazer consigo vieses em relação a eventuais posicionamentos. O estudo se limitou, ainda, a analisar uma crise ainda em curso, sugerindo-se uma análise posterior da efetividade das respostas organizacionais. Além disso, analisar os processos na estrutura organizacional nos diferentes momentos da pandemia e o impacto dos atributos organizacionais na efetividade das diferentes respostas ao longo da crise, bem como os impactos dessas respostas na estrutura do campo, podem se apresentar como uma agenda promissora.

\section{REFERÊNCIAS}

ABREU, M. C. S.; ANDRADE, R. J. C. Dealing with wicked problems in socio-ecological systems affected by industrial disasters: A framework for collaborative and adaptive governance.

Science of The Total Environment, v. 694, p. 133700, 2019. DOI:

https://doi.org/10.1016/j.scitotenv.2019.133700.

BARDIN L. L. Analyse de contenu. Editora: Presses Universitaires de France, 1977.

CAMARGO, B. V.; JUSTO, A. M. IRAMUTEQ: Um software gratuito para análise de dados textuais. Temas em Psicologia, 2013.

DACIN, M. T.; MUNIR, K.; TRACEY, P. Formal dining at Cambridge colleges: Linking ritual performance and institutional maintenance. Academy of Management Journal, v. 53, n. 6, p. 1393-1418, 2010. DOI: https://doi.org/10.5465/AMJ.2010.57318388.

DIMAGGIO, P. J.; POWELL, W. W. The Iron Cage Revisited: Institutional Isomorphism and Collective Rationality in Organizational Fields. American Sociological Review, v. 48, n. 2, p. 147-160, 1983. DOI: https://doi.org/10.2307/2095101.

DUBOIS, A.; GADDE, L. E. Systematic combining: An abductive approach to case research. Journal of Business Research, v. 55, n. 7, p. 553-560, 2002. DOI: 10.1016/S01482963(00)00195-8.

DURAND, R. Advancing strategy and organization research in concert: Towards an integrated model? Strategic Organization, v. 10, n. 3, p. 297-303, 2012. DOI:

10.1177/1476127012453290.

FOLKE, C. Resilience: The emergence of a perspective for social-ecological systems analyses. Global Environmental Change, v. 16, n. 3, p. 253-267, 2006. DOI:

10.1016/j.gloenvcha.2006.04.002.

FRIEDLAND, R.; ALFORD, R. R. Bringing Society Back In: Symbols, Practices and Institutional Contradictions. In: POWELL, W. W.; DIMAGGIO, P. J. (Eds.). . The New Institutionalism in Organizational Analysis. Chicago: University of Chicago Press, 1991. p. 232-263.

GREENWOOD, R.; RAYNARD, M.; KODEIH, F.; MICELOTTA, E. R.; LOUNSBURY, M. Institutional Complexity and Organizational Responses. The Academy of Management Annals, v. 5, n. 1, p. 317-371, 2011. DOI: 10.1080/19416520.2011.590299. 
HERNANTES, J.; BAÑULS, V. A. Moving forward to disaster resilience: Perspectives on increasing resilience for future disasters. Technological Forecasting and Social Change, $v$. 121, p. 1-6, 2017. DOI: 10.1016/j.techfore.2017.05.011.

KRUMKAMP, R.; AHMAD, A.; KASSEN, A.; HJARNOE, L.; SYED, A. M.; ARO, A. R.; REINTJES, R. Evaluation of national pandemic management policies-A hazard analysis of critical control points approach. Health Policy, v. 92, n. 1, p. 21-26, 2009. DOI:

10.1016/j.healthpol.2009.01.006.

KUHN, T. S. The Structure of Scientific Revolutions. [s.l: s.n.].

MEYER, J.; SCOTT, W. R.; STRANG, D. Centralization, Fragmentation, and School District Complexity. Administrative Science Quarterly, v. 32, n. 2, p. 186-201, 1987. DOI: https://doi.org/10.2307/2393125.

MEYER, J. W.; ROWAN, B. Institutionalized Organizations: Formal Structure as Myth and Ceremony. American Journal of Sociology, v. 83, n. 2, p. 340-363, 1977.

PACHE, A. C.; SANTOS, F. When worlds collide: The internal dynamics of organizational responses to conflicting institutional demands. Academy of Management Review, v. 35, n. 3, p. 455-476, 2010. DOI: 10.5465/amr.35.3.zok455.

ROBERT, N. Wicked Problems and Network Approaches to Resolution. International Public Management Review, v. 1, n. 1, p. 1-19, 2000.

ROSENSCHÖLD, J. M.; ROZEMA, J. G.; FRYE-LEVINE, L. A. Institutional inertia and climate change: A review of the new institutionalist literature. Wiley Interdisciplinary Reviews: Climate Change, v. 5, n. 5, p. 639-648, 2014. DOI: 10.1002/wcc.292.

SALVIATI, M. E. Manual do aplicativo IRaMuTeQ. Disponível em: http://www.iramuteq.org/documentation/html. Acesso em: 10 abril. 2020

SANDER, B.; NIZAM, A.; GARRISON JR, L. P.; POSTMA, M. J.; HALLORAN, M. E.; LONGINI JR, I. $M$. Economic evaluation of influenza pandemic mitigation strategies in the United States using a stochastic microsimulation transmission model. Value in Health, v. 12, n. 2, p. 226233, 2009. DOI: https://doi.org/10.1111/j.1524-4733.2008.00437.x.

THORNTON, P. H.; OCASIO, W. Institutional Logics and the Historical Contingency of Power in Organizations : Executive Succession in the Higher Education Publishing Industry, 1958 1990. American Jounal of Sociology, v. 105, n. 3, p. 801-843, 1999. DOI:

https://doi.org/10.1086/210361.

THORNTON, P. H.; OCASIO, W. Institutional Logics. In: POWELL, W. W.; DIMAGGIO, P. J. (Eds.). . The SAGE Handbook of Organizational Institutionalism. Chicago: University of Chicago Press, 2008. p. 99-128. DOI: 10.4135/9781849200387.n4.

WEBER, E. P.; KHADEMIAN, A. Wicked Problems, Knowledge Challenges \& Collab Capacity in Network Settings. Public Administration Review, v. 68, n. 2, p. 334-349, 2008. DOI: 10.1111/j.1540-6210.2007.00866.x.

ZUCKER, L. G. The Role of Institutionalization in Cultural Persistence. American Sociological Review, v. 42, n. 5, p. 726-743, 1977. DOI: https://doi.org/10.2307/2094862. 